\title{
Three-dimensional muscle architecture of the infant and adult trapezius: a cadaveric study
}

\author{
Mikaela L. Stiver ${ }^{1,2}$ (D), Luke R. Bradshaw ${ }^{3}$ (D), Ethan M. Breinhorst ${ }^{3}$ (D), Anne M. R. Agur ${ }^{1,2}$ (D), \\ S. Ali Mirjalili ${ }^{3}$ \\ ${ }^{1}$ Division of Anatomy, Department of Surgery, Faculty of Medicine, University of Toronto, Toronto, Ontario, Canada \\ ${ }^{2}$ Rehabilitation Sciences Institute, Faculty of Medicine, University of Toronto, Toronto, Ontario, Canada \\ ${ }^{3}$ Department of Anatomy and Medical Imaging, University of Auckland, Auckland, New Zealand
}

\begin{abstract}
Objectives: The elaborate morphometry of the human trapezius muscle facilitates its involvement in numerous active movements of the shoulder girdle and passive stabilization of the upper extremity. Despite its functional importance throughout the lifespan, little is known about the 3D architecture of trapezius at any post-natal timepoints. Accordingly, the aim of this preliminary cadaveric study was to digitize, quantify, model, and compare the 3D architecture of trapezius at two temporal extremes: infancy and adulthood.

Methods: We examined trapezius in two female formalin-embalmed cadavers, aged 6 months and 72 years, respectively. We meticulously dissected each muscle, allowing us to digitize and model the comprehensive muscle architecture in situ at the fiber bundle level. We quantified standard architectural parameters to facilitate comparison between each functional partition of trapezius (i.e., descending, transverse, ascending) and proportionally between the infant and adult specimens.

Results: We found markedly different patterns in fiber bundle length range, physiological cross-sectional area, and muscle volume within and between muscles. Notably, the proportional physiological cross-sectional area of the ascending and descending partitions was equal (1:1) in the infant, in contrast to 3:1 in the adult. The transverse partitions were proportionally similar, accounting for over half of the whole muscle physiological cross-sectional area in both specimens.

Conclusion: This study provides preliminary insights into infant and adult trapezius architecture at an unparalleled level of detail and precision. The quantifiable architectural differences appear to coincide with functional development-a notion that warrants further investigation in larger samples and with longitudinal approaches.
\end{abstract}

Keywords: cadaver; digitization; infant; muscle architecture; skeletal muscle; trapezius

Anatomy 2021;15(1):26-35 @2021 Turkish Society of Anatomy and Clinical Anatomy (TSACA)

\section{Introduction}

The trapezius is a morphometrically complex muscle that is conventionally divided into three distinct partitions: ascending, transverse, and descending. Functionally, the trapezius is involved in voluntary movement of the scapulae (depression, retraction, elevation, and upward rotation) and clavicles (elevation), as well as postural stability and upper extremity support via suspension of the shoulder girdle. ${ }^{[1]}$ This large superficial muscle is frequently implicated in a wide range of pathologic conditions, including fibromyalgia, ${ }^{[2,3]}$ cerebral palsy, ${ }^{[4]}$ and facioscapulohumeral muscular dystrophy. ${ }^{[5]}$ Morphological changes present in these conditions include reduction of muscle mass due to atrophy leading to functional changes such as altered excursion (range of motion) or reduced relative force generating capacity. ${ }^{[6]}$

Despite its common involvement in pathologic conditions, very little is known about both the detailed architecture of the trapezius and its developmental trajectory. Without this foundational knowledge, assessment of the

This study was presented as an oral presentation at the 36th Annual Meeting of the American Association of Clinical Anatomists, 7une 11th-15th 2019, Tulsa, OK, USA. 
etiology, pathophysiology, rehabilitation, and management of trapezius pathologies are inherently limited.

A recent study by Badura et al ${ }^{\left[{ }^{[7]}\right.}$ reported preliminary quantitative data regarding pre-natal growth dynamics of the human trapezius. This study offers vital insights into fetal trapezius development; however, fetal development patterns are not influenced by external functional demands and therefore cannot be generalized to postnatal growth. Post-natal data are essential for understanding how functionality may affect the morphometry and growth dynamics of muscles like the trapezius.

In early post-natal development, the trapezius (in tandem with the sternocleidomastoid) generates a head thrust movement that is essential to infant breastfeeding mechanics. ${ }^{[8]}$ Moreover, it has been demonstrated that stabilization of the head and shoulder are facilitated by increases in trapezius and deltoid activity. These muscular changes provide increased postural stability to enable the development of functional reaching patterns. ${ }^{[9]}$ Nevertheless, to the best of our knowledge, no studies to date have investigated or documented the morphometry of the infant trapezius.

The adult trapezius has been examined in greater detail than that of the infant. Numerous in vivo imaging studies (e.g., ultrasound and magnetic resonance) have been used to quantify various morphometric parameters, including cross-sectional area, fiber bundle length (FBL), and muscle thickness. ${ }^{[10-13]}$ Yet, a lack of thorough understanding of the fundamental anatomy in this region poses a significant challenge to imaging optimization and interpretation. Several cadaveric studies have investigated adult trapezius morphometry; ${ }^{[14-17]}$ however, the architectural parameters quantified in these studies are often overgeneralized and inaccurate due to limited fiber bundle (FB) sampling, lack of volumetric data, and 2D manual measurements of 3D parameters. Recently, our lab has begun digitizing the 3D architecture of the adult trapezius in cadaveric specimens using the same methods presented in this paper ${ }^{[18]}$ however, data analysis is still in progress.

Accordingly, this study had two primary objectives; (1) to capture and quantify three-dimensional (3D) trapezius architecture in an infant and adult cadaveric specimen, and (2) to document morphometric differences between the infant and adult trapezius.

\section{Materials and Methods}

This study examined two lightly formalin-embalmed cadaveric specimens: one female infant (age: 6 months) and one female adult (age: 72 years). Neither specimen had discernable evidence of structural abnormality, pathology, or previous surgery in the back, neck, or shoulder regions.

The digitization and modeling techniques used in this study were developed in our laboratory and have been used previously to examine the morphometry of other muscles, including supraspinatus, ${ }^{[19]}$ pectoralis major, ${ }^{[20]}$ and soleus. ${ }^{[21]}$

Prior to data collection, we obtained full body computerized tomography (CT) scans of the infant cadaver on an Aquilion ONE ${ }^{\mathrm{TM}} 320$ scanner (Toshiba Medical Systems Corporation, Tokyo, Japan) with a voxel size of $0.62 \mathrm{~mm} \times$ $0.62 \mathrm{~mm} \times 0.3 \mathrm{~mm}$. While the bony attachments of the adult trapezius have been studied in detail, no studies to our knowledge have characterized the attachments of the infant trapezius with respect to the progressive ossification of bones such as the scapula and clavicle. Next, each cadaver was stabilized in a prone position with the scapulae fixed in a neutral position against the rib cage. In the adult specimen, both humeri were in anatomical position, while in the infant the right humerus was slightly abducted $\left(<30^{\circ}\right)$. Only the right trapezius was studied in the infant specimen due to positional limitations of the left upper extremity resulting from the tissue fixation process; both the left and right trapezius were analyzed in the adult specimen.

The skin and subcutaneous tissues were carefully removed to expose each trapezius muscle in its entirety. Beginning on the superficial surface of the trapezius, each FB was meticulously delineated in situ between its lateral and medial attachment sites (Figure 1). FBs were digitized in 3-5 $\mathrm{mm}$ increments, from origin to insertion, using a MicroScribe ${ }^{\circledast} \mathrm{G}$ digitizer (Immersion Corp.; San Jose, CA, USA). Digitized FBs were then removed to reveal underlying FBs, and the process of serial digitization and dissection was repeated through the entire volume of the muscle. The surfaces of aponeuroses that provided attachment for FBs to bone were digitized in a grid pattern as they were exposed during dissection. The resultant point cloud data were segmented into the three functional partitions of the trapezius based on individual FB attachments to lateral bony landmarks examined in 3D: ascending partitionFBs with lateral attachment to the base of the scapular spine (end of the deltoid tubercle); transverse partitionFBs with lateral attachment to the scapular spine or acromion; and descending partition-FBs with lateral attachment to the clavicle. Subsequently, the data were reconstructed as 3D models of the connective and contractile elements of each trapezius using Autodesk ${ }^{\circledR}$ Maya ${ }^{\circledR}$ 2019 (Autodesk Inc.; San Rafael, CA, USA).

Digitized data were imported into a custom program developed in the laboratory to quantify FBL, FBL range, 

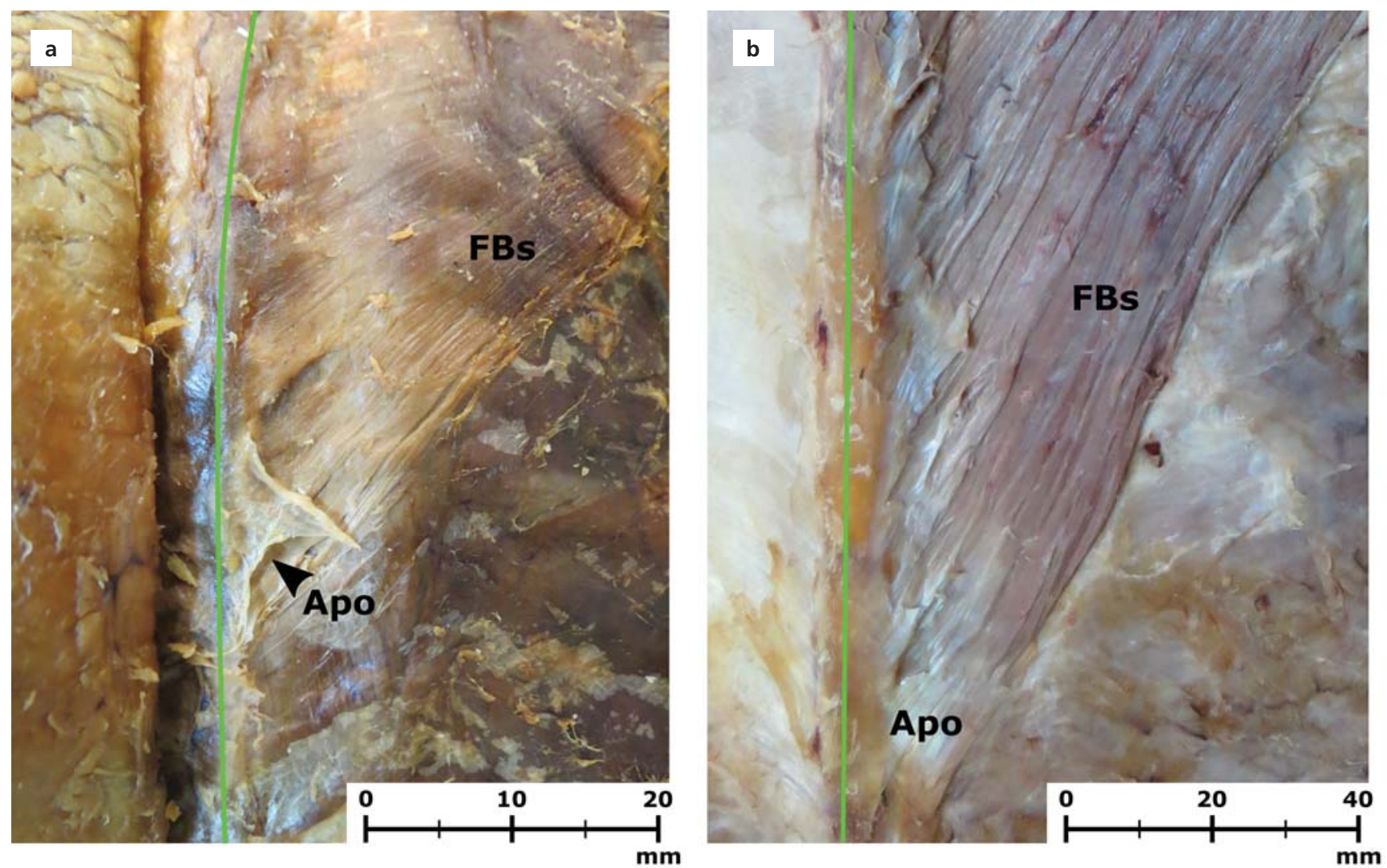

Figure 1. Dissection photographs of fiber bundles in the ascending partition of the infant (a) and adult (b) specimens. The green line shows the spinous processes of the thoracic vertebrae. Apo: Aponeurosis; FBs: fiber bundles.

muscle volume (MV), and physiological cross-sectional area (PCSA) for each functional partition according to the method described in Lee et al. ${ }^{[2]}$ Each muscle parameter is defined as follows: (a) FBL: the length of a single FB between its attachment sites and is a measure of excursion; (b) MV: the volume of a muscle or partitions thereof and is a component of PCSA; and (c) PCSA: MV accounting for the internal architecture of a muscle and represents relative force generating capability of a muscle or a functional partition. Proportional values were also calculated for PCSA and MV (\% of whole muscle) and FBL range (\% of whole muscle range) to facilitate comparison between muscles.

Descriptive statistics (mean, standard deviation, range) were calculated for FBL. One-way analysis of variance (ANOVA) was conducted to assess significant differences in FBL between functional partitions for each trapezius. All statistical analyses were conducted in SPSS ${ }^{\circledR}$ Statistics v.26.0 (IBM ${ }^{\oplus}$; Armonk, NY, USA) using a significance level of $\alpha=0.05$. If a one-way ANOVA demonstrated statistical significance, data were tested for normality and homogeneity of variance and appropriate post-hoc inde- pendent-samples t-tests were used to explore differences. Significance levels were adjusted using the Bonferroni correction where appropriate. Relative PCSA and MV for each partition were compared between the infant and adult muscles.

\section{Results}

Contractile tissues of the trapezius in the infant and adult spanned between wide, flat connective tissue elements (i.e., aponeuroses) both medially and laterally. These aponeuroses were more extensive in the adult, attaching medially to the thoracic spinous processes, nuchal ligament, and superior nuchal line and laterally to the scapular spine, acromion, and lateral part of the clavicle. In the infant these tissues were less developed, and some bony features were still cartilaginous, as seen in the CT scans onto which the infant trapezius model was superimposed (Figure 2). We also found a rhomboidal aponeurosis extending inferomedially from the base of the scapular spine in both the infant and adult specimens. 


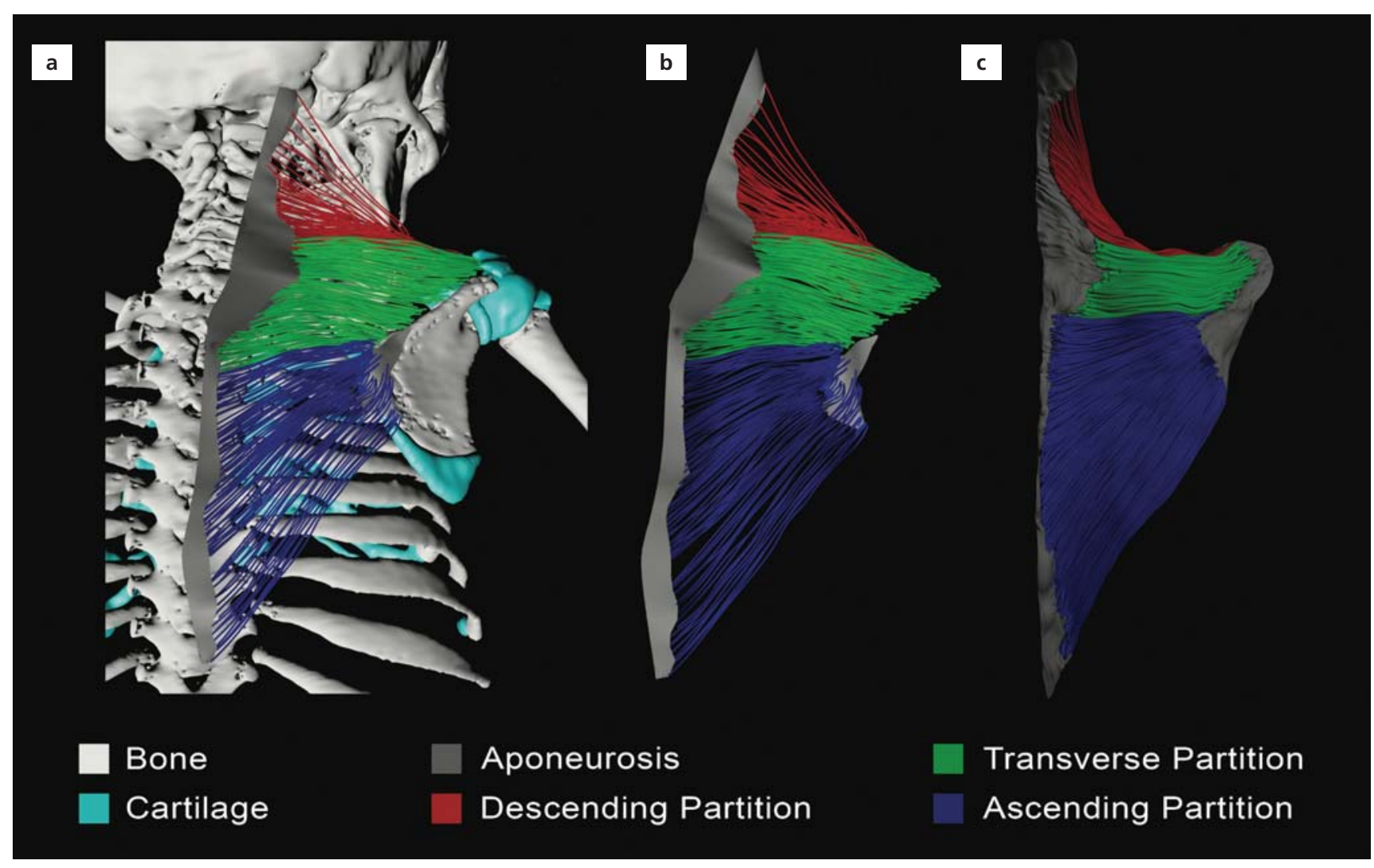

Figure 2. Three-dimensional models of the infant and adult trapezius rendered in Autodesk ${ }^{\oplus}$ Maya ${ }^{\circledast}$ from the digitized cadaveric data. (a) Right infant trapezius registered onto computerized tomography-scanned skeletal and cartilaginous elements; (b) right infant trapezius; and (c) right adult trapezius.

\section{Infant Trapezius Architecture}

The infant trapezius had a mean FBL of $37.64 \pm 6.72 \mathrm{~mm}$. Each functional partition (descending, transverse, and ascending) exhibited distinct morphometric characteristics (Figure 3). The ascending partition had the largest range of FBLs and the transverse partition had the shortest average FBL (Table 1).

A one-way ANOVA revealed significant differences in FBL between partitions in the infant trapezius $\left(\mathrm{F}_{(2,313)=}\right.$ 58.700, $\mathrm{p}<0.001)$. Post hoc independent-samples t-tests indicated that the mean FBL in the transverse partition was significantly smaller than in both the descending partition $(\mathrm{t}=13.096, \quad \mathrm{p}<0.001 ; 95 \%$ confidence interval [CI]: $6.23-8.43)$ and the ascending partition $(\mathrm{t}=7.594, \mathrm{p}<0.001$; 95\% CI: 5.07-8.63). There was no significant difference between the descending and ascending FBL $(\mathrm{p}=0.601)$.

The PCSA for the infant trapezius was $147.88 \mathrm{~mm}^{2}$ and the MV was $7.51 \mathrm{~cm}^{3}$ (Table 1). In the infant, the proportional PCSA and MV values differed markedly in the descending and transverse partitions. Conversely, the transverse partition had a the largest proportional PCSA, but a similar MV to the descending partition. The relative PCSA and MV in the ascending partition were almost identical (Figure 4).

\section{Adult Trapezius Architecture}

The left adult trapezius had a mean FBL of $93.06 \pm 25.01$ $\mathrm{mm}$. As with the infant, functional partitions of the adult

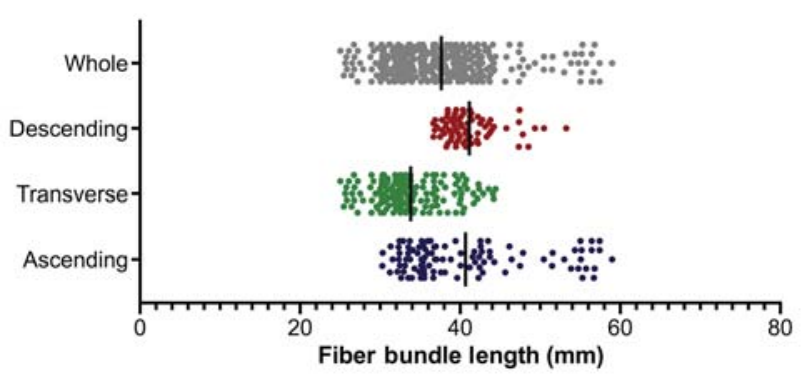

Figure 3. Infant trapezius: fiber bundle lengths. Each point represents the length of one fiber bundle in the whole muscle and each functional partition. The vertical black line indicates the mean fiber bundle length Additional numerical details are provided in Table 1. 
Table 1

Architectural parameters: infant trapezius

\begin{tabular}{lcccccccc} 
Muscle partition & $\mathbf{n}$ & $\begin{array}{c}\text { Mean FBL+SD } \\
(\mathbf{m m})\end{array}$ & $\begin{array}{c}\text { FBL range } \\
(\text { Min.-max.) }\end{array}$ & $\begin{array}{c}\text { \% Whole } \\
\text { FBL range }\end{array}$ & $\begin{array}{c}\text { PCSA } \\
\left(\mathbf{m m}^{2}\right)\end{array}$ & $\begin{array}{c}\text { \% Whole } \\
\text { PCSA }\end{array}$ & $\begin{array}{c}\text { MV } \\
\left(\mathbf{c m}^{\mathbf{3}}\right)\end{array}$ & $\begin{array}{c}\text { \% Whole } \\
\mathbf{M V}\end{array}$ \\
\hline Whole & 314 & $37.64 \pm 6.72$ & $25.04-58.98$ & - & 147.88 & - & 7.51 & - \\
Descending & 71 & $41.14 \pm 3.46$ & $36.65-53.25$ & $48.91 \%$ & 31.89 & $21.25 \%$ & 2.90 & $38.66 \%$ \\
Transverse & 144 & $33.82 \pm 4.53$ & $25.04-44.41$ & $57.08 \%$ & 85.11 & $57.56 \%$ & 2.98 & $39.68 \%$ \\
Ascending & 99 & $40.66 \pm 8.15$ & $30.28-58.98$ & $84.55 \%$ & 31.34 & $21.19 \%$ & 1.63 & $21.65 \%$ \\
\hline
\end{tabular}

FBL: fiber bundle length; MV: muscle volume; n: number of fiber bundles; PCSA: physiological cross-sectional area; SD: standard deviation.

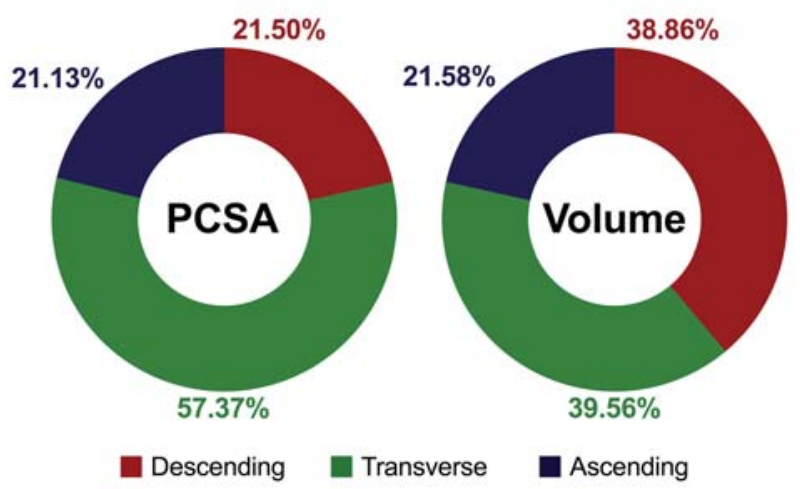

Figure 4. Infant trapezius: proportional physiological cross-sectional area and muscle volume. This figure illustrates the proportional physiological cross-sectional area (labeled: PCSA) and muscle volume (labeled: Volume) for each functional partition of the right infant trapezius. The percentage of whole muscle values are printed around each chart in the corresponding color for each partition. Additional numerical details are provided in Table 1.

trapezius muscles were morphometrically distinct (Figure 5). The ascending partition included both the shortest and longest FBLs in the muscle while the transverse partition had both the shortest mean FBL and the smallest range of FBLs (Table 2).

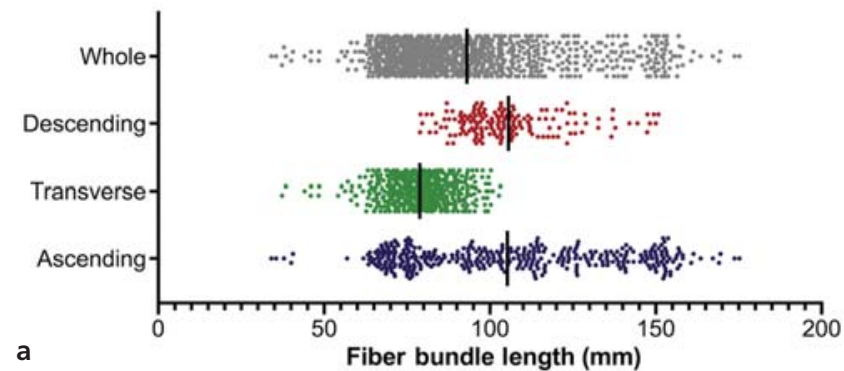

The right trapezius was slightly smaller than the left but had a similar range of FBLs. The mean FBLs for each partition exhibited a similar pattern to those in left trapezius. As found in the left trapezius, the ascending partition of the right trapezius also included $100 \%$ of the range of FBLs documented in the whole muscle (Table 2).

One-way ANOVAs for both left and right adult muscles indicated significant differences between partitions

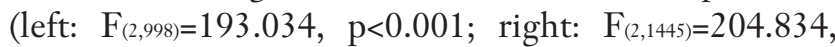
$\mathrm{p}<0.001)$. Post hoc independent-samples t-tests of the left trapezius revealed that the mean FBL in the transverse partition was significantly smaller than in the descending partition ( $\mathrm{t}=20.157, \mathrm{p}<0.001$; $95 \%$ CI: $24.10-29.32)$ and in the ascending partition $(\mathrm{t}=15.634, \mathrm{p}<0.001 ; 95 \%$ CI: 23.05 29.68). Average FBLs in the ascending and descending partitions were not significantly different in the left trapezius ( $p=0.864)$. Analyses of the right trapezius revealed the same differences between the descending and transverse mean FBLs ( $t=18.779, \mathrm{p}<0.001 ; 95 \%$ CI: $18.73-23.13)$ as well as between the ascending and transverse mean FBLs $(\mathrm{t}=17.849, \mathrm{p}<0.001$; 95\% CI: 24.29-30.30); however, the ascending and descending partition average FBLs were also significantly different ( $\mathrm{t}=3.486$, $\mathrm{p}=0.001$; $95 \%$ CI: 2.78 9.95).

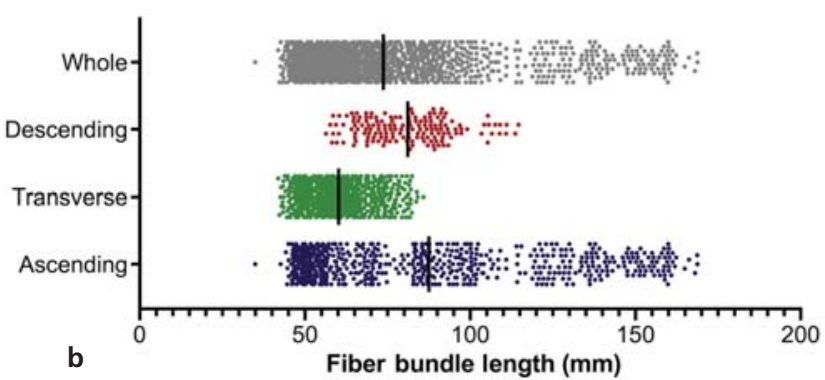

Figure 5. Adult trapezius: fiber bundle lengths. Each point represents the length of one fiber bundle in the whole muscle and each functional partition of the left trapezius (a) and right trapezius (b). The vertical black line indicates the mean fiber bundle length. Additional numerical details are provided in Table 2 . 
Table 2

Architectural parameters: adult trapezius.

\begin{tabular}{|c|c|c|c|c|c|c|c|c|}
\hline Muscle partition & n & $\begin{array}{l}\text { Mean FBL } \pm \text { SD } \\
(\mathrm{mm})\end{array}$ & $\begin{array}{l}\text { FBL range } \\
\text { (Min.-max.) }\end{array}$ & $\begin{array}{l}\% \text { Whole } \\
\text { FBL range }\end{array}$ & $\begin{array}{l}\text { PCSA } \\
\left(\mathrm{mm}^{2}\right)\end{array}$ & $\begin{array}{c}\% \text { Whole } \\
\text { PCSA }\end{array}$ & $\begin{array}{l}\text { MV } \\
\left(\mathrm{cm}^{3}\right)\end{array}$ & $\begin{array}{c}\text { \% Whole } \\
\text { MV }\end{array}$ \\
\hline Whole: Left & 1001 & $93.06 \pm 25.01$ & $34.12-175.23$ & - & 811.77 & - & 79.48 & - \\
\hline Descending & 164 & $105.60 \pm 15.83$ & $78.90-150.80$ & $50.95 \%$ & 95.25 & $11.73 \%$ & 11.15 & $14.03 \%$ \\
\hline Transverse & 465 & $78.89 \pm 10.30$ & 37.34-103.16 & $46.64 \%$ & 408.12 & $50.28 \%$ & 33.21 & $41.78 \%$ \\
\hline Ascending & 372 & $105.25 \pm 31.19$ & $34.12-175.23$ & $100.00 \%$ & 308.40 & $37.99 \%$ & 35.13 & $44.20 \%$ \\
\hline Whole: Right & 1448 & $73.75 \pm 27.90$ & $34.97-168.80$ & - & 874.13 & - & 73.34 & - \\
\hline Descending & 157 & $81.14 \pm 13.25$ & $56.36-114.66$ & $43.56 \%$ & 94.58 & $10.82 \%$ & 13.01 & $17.73 \%$ \\
\hline Transverse & 693 & $60.21 \pm 9.26$ & $41.93-85.87$ & $32.83 \%$ & 481.41 & $55.07 \%$ & 31.30 & $42.68 \%$ \\
\hline Ascending & 598 & $87.50 \pm 36.40$ & $34.97-168.80$ & $100.00 \%$ & 298.13 & $34.11 \%$ & 29.03 & $39.59 \%$ \\
\hline
\end{tabular}

FBL: fiber bundle length; MV: muscle volume; n: number of fiber bundles; PCSA: physiological cross-sectional area; SD: standard deviation.

The PCSAs for the left and right adult trapezius muscle were 811.77 and $874.13 \mathrm{~mm}^{2}$, respectively (Table 2). In both adult muscles, the transverse partitions accounted for over half of the whole muscle PCSA while the proportional MVs of this partition were slightly lower. In contrast, the proportional PCSA was lower than the proportional MV in both the descending and ascending partitions (Figure 6).

\section{Morphometric Comparison}

On the surface, the infant and adult trapezius were fairly similar in terms of shape and attachment to skeletal elements. Some bony elements (e.g., acromion) were not yet fully developed in the infant and were instead cartilaginous in composition. Morphometrically, the variability in FBL throughout the muscle, and within each functional partition, was comparable between the infant and adult muscles. The FBL range in the descending partitions were just under half of the whole muscle range $(48.91 \%$ in the infant vs $47.26 \%$ in the adult). The ascending partitions had the largest range of FBLs in both the infant and adult muscles, while the transverse partitions had the smallest range of FBLs (Table 3).

The trapezius in infancy and adulthood exhibited several other notable disparities. The infant trapezius MV was about one tenth that of the adult trapezius. MV was evenly distributed between the descending and transverse partitions in the infant, with the ascending partition being markedly smaller. In the adult, nearly $85 \%$ of the $M V$ was divided between the transverse and ascending partitions, while the descending partition was strikingly smaller (Table 3).

Compared to the infant trapezius PCSA, the average adult muscle PCSA was 5.7 times greater. The transverse
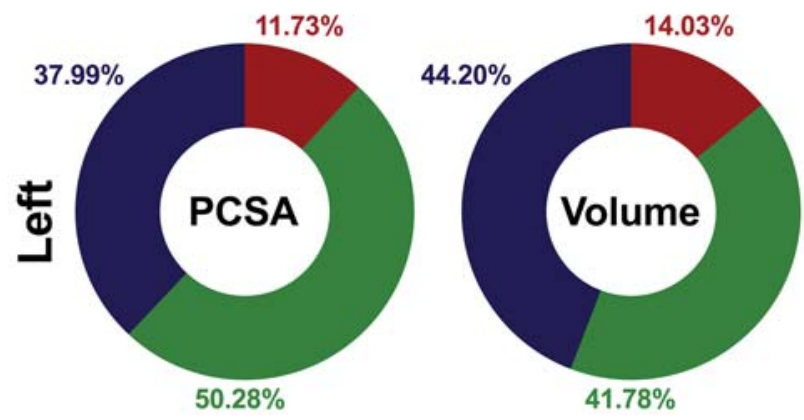
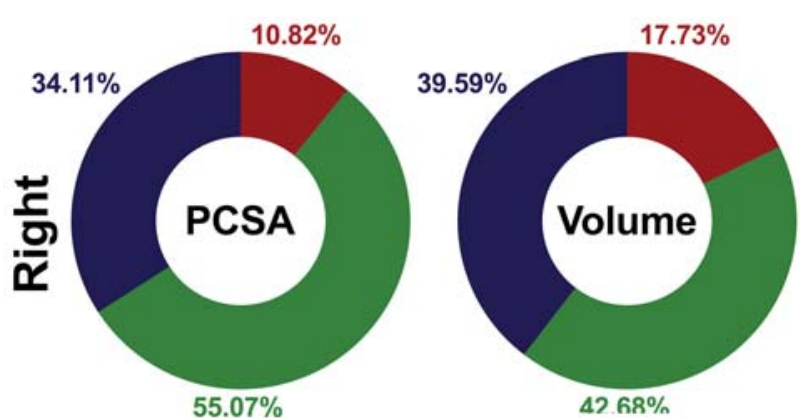

Descending

Transverse

Ascending

Figure 6. Adult trapezius: proportional physiological cross-sectional area and muscle volume. This figure illustrates the proportional physiological cross-sectional area (labeled: PCSA) and muscle volume (labeled: Volume) for each functional partition of the left and right adult trapezius. The percentage of whole muscle values are printed around each chart in the corresponding color for each partition. Additional numerical details are provided in Table 2 .

partitions of the infant and adult muscles were proportionally similar, accounting for just over half of the whole muscle PCSA. In the infant, PCSA was equally distributed between the ascending and descending partitions; on the contrary, the ascending partition PCSA in the adult was 
Table 3

Morphometric changes: infancy to adulthood.

\begin{tabular}{|c|c|c|c|c|c|c|}
\hline \multirow[b]{2}{*}{ Muscle partition } & \multicolumn{2}{|c|}{$\%$ Whole FBL range } & \multicolumn{2}{|c|}{$\%$ Whole PCSA } & \multicolumn{2}{|c|}{ \% Whole MV } \\
\hline & Infant & Adult & Infant & Adult & Infant & Adult \\
\hline Descending & $48.91 \%$ & $47.26 \%$ & $21.25 \%$ & $11.28 \%$ & $38.66 \%$ & $15.88 \%$ \\
\hline Transverse & $57.08 \%$ & $39.74 \%$ & $57.56 \%$ & $52.68 \%$ & $39.68 \%$ & $42.23 \%$ \\
\hline Ascending & $84.55 \%$ & $100 \%$ & $21.19 \%$ & $36.05 \%$ & $21.65 \%$ & $41.90 \%$ \\
\hline
\end{tabular}

FBL: fiber bundle length; MV: muscle volume; PCSA: physiological cross-sectional area.

approximately three times greater than that of the descending partition (Table 3).

The proportional adult MV and PCSA were comparable, with a slightly higher MV than PCSA in the descending and ascending partitions and vice versa in the transverse partition. Proportional MV and PCSA in the infant trapezius partitions were noticeably incongruent. For example, while the descending and transverse partitions had almost identical proportional MVs, the PCSA in the transverse partition was over $170 \%$ greater than the descending partition PCSA.

\section{Discussion}

To the best of our knowledge, this is the first study to document and quantify the comprehensive 3D architecture throughout the muscle volume of the infant and adult trapezius. The preliminary results presented in this paper offer an unprecedented level of precision and detail regarding the trapezius muscle. Likewise, our findings support the long-held pediatric dogma that, from the perspective of muscle architecture, "children are not little adults".

\section{Trapezius Morphology}

One of the major confounds in musculoskeletal literature involving trapezius is a lack of consensus regarding the precise definitions of anatomical or functional partitions within the muscle. While most sources divide trapezius into three partitions-ascending, transverse, and descending-there are inconsistencies in how each partition is defined. In particular, FBs that attach laterally to the medial border of the acromion are variably included in either the descending partition ${ }^{[23]}$ or the transverse partition. ${ }^{[24,25]}$ Several electromyographical studies have divided trapezius into four partitions; ${ }^{[26,27]}$ however, there is currently no clear evidence to support this further subdivision. The partitioning of trapezius is believed to have been derived from non-human literature, ${ }^{[28]}$ further substantiat- ing the need for detailed studies of this muscle in humans. As a result, it is currently challenging to compare between studies in which different criteria have been used to partition trapezius.

The broad morphological characteristics of trapezius observed in this study are mostly in agreement with those presented in the existing cadaveric literature. Similar to Kamibayashi and Richmond, ${ }^{[16]}$ we observed that the FBs throughout the muscle volume span the full width of the muscle, from medial origin to lateral insertion. We also noted a similar expansive aponeurosis extending laterally from the spinous processes in the transverse partition; however, in the infant there were no markedly shorter FBs present in the deep transverse partition, and it lacked the substantial thickness seen in the adult. The common aponeurotic tendon attaching to the base of the scapular spine was consistent with that described by Johnson et al. ${ }^{[15]} \mathrm{We}$ observed that all FBs in the ascending partition of both the infant and adult trapezius inserted onto this rhomboidal aponeurosis, in contrast to Johnston et al. ${ }^{[15]}$ in which this aponeurosis was described as the lateral attachment only for fascicles originating from T2 through T5. Similar levels of qualitative detail regarding trapezius morphometry were not reported in other cadaveric literature, and we did not find any non-adult cadaveric studies including trapezius.

\section{Muscle Architecture}

We also compared the quantitative results of our pilot study to existing adult literature; there were no quantitative studies of pediatric trapezius architecture with which to compare our findings. Of the four cadaveric studies of adult trapezius architecture that included numerical data, ${ }^{[15-17,29]}$ only one presented data divided into three distinct muscle partitions. In general, the proportional relationships reported by Kamibayashi and Richmond ${ }^{[16]}$ are similar to those in our adult cadaver: the ascending partition had the largest average FBL of the three partitions (right muscle only in the current study)—including both 
the shortest and longest FBLs recorded in the whole muscle, the descending partition PCSA was the smallest, and the transverse partition PCSA was the largest. In the current study, the FBL values were slightly smaller (e.g., overall FBL range of $34.12-175.23 \mathrm{~mm}$ compared to $56.0-225.0 \mathrm{~mm}$ [converted from $\mathrm{cm}$ ]), as were the PCSA values. These differences may be attributable to numerous factors including donor height and weight, measurement methodology, and FB sampling. In the current study, every FB throughout the muscle volume is included in the dataset, compared to a mere 5-20 FBs pseudo-randomly sampled from each muscle.

A more extensive literature has been published on the adult trapezius using in vivo magnetic resonance imaging and ultrasound; however, the majority of these studies are focused on pathological conditions and rarely include values for baseline or asymptomatic trapezius architectural parameters. Those that do typically report parameters with limited functional value-such as muscle thickness, ${ }^{[1,29,30]} \mathrm{MV}{ }^{[31]}$ or anatomical cross-sectional area (i.e., measured in standard anatomical planes) ${ }^{[10,11,31]}$ —as opposed to FBL or PCSA. Compared to the adult MVs calculated in the current study, the trapezius MVs reported by Li et al. ${ }^{[31]}$ from serial MRI scans were much larger, ranging from $170-530 \mathrm{~cm}^{3}$; however, this is consistent with a study that reported MV estimated from MRI were an average of $64 \%$ larger than those estimated from cadaveric dissection. ${ }^{[2]]}$ Nonetheless, studies suggest that FBL does not change as long as tissue is fixed while remaining intact with bony attachments ${ }^{[32]}$ and that relative or proportionate values for PCSA are consistent between computed tomography images and cadaveric data. ${ }^{[33]}$

\section{Functional Implications}

Pre-natal data, such as those reported by Badura et al., ${ }^{[7]}$ provide insight into fetal muscle developmental trajectories. Nevertheless, pre-natal muscle growth is not influenced by the same types of external forces and functional demands present during post-natal development and adulthood. The morphometric differences between the infant and adult trapezius muscles documented in this study suggest that external forces and functional demands during post-natal development change the muscle architecture in favour of postural stability and functional upper limb movement.

At six months of post-natal age, an infant is typically able to elevate their head and chest off the ground by extending their arms from a prone position and may begin to maneuver themselves along the floor with their arms and legs. ${ }^{[34]}$ These developmental milestones are consistent with the patterns of PCSA (relative force generating capability) observed within the infant trapezius: the transverse partition-which functionally is associated with scapular retraction and, to a lesser extent, depression-accounts for over half of the whole muscle PCSA. These scapular movements are integral to the sequential activity of raising the head and chest in a prone position using the upper limbs. Without scapular retraction and depression, this functional task would not be possible.

The remaining PCSA is equally distributed between the ascending and descending partitions of the trapezius, in stark contrast to the approximate 3:1 ratio seen in the adult. This may be explained by the considerable increases in muscle tone required during the development of voluntary head and neck movement during early infancy, such as the head thrust movement required for productive breastfeeding. When the scapulae are stabilized, the descending partition of trapezius assists with head/neck extension, ipsilateral flexion, and contralateral rotation. ${ }^{[25]}$ In the adult, the ascending partition may have greater force generating capabilities due to functional demands associated with upright posture and positioning of the upper limb; in contrast, the descending partition may remain more functionally analogous to that of the infant in terms of gross motor activity, resulting in the unbalanced PCSA ratio observed in the adult.

Furthermore, electromyographical studies of the adult trapezius have reported that "the greatest activity in trapezius appears during abduction of the limb and chiefly in the lower two-thirds of the muscle". ${ }^{[3]}$ This is in keeping with our findings that the ascending and transverse partitions jointly contained nearly $90 \%$ of the whole muscle PCSA and $85 \%$ of the whole MV.

\section{Limitations and Strengths}

The most notable limitation of this study is the small sample size: one infant and one adult. This is largely attributable to strict regulations regarding non-adult body donation resulting in a dearth of cadaveric data during key developmental timepoints. Adult data is more widely available; however, due to the preliminary nature of this study we chose to include data from a single female adult cadaver for the purposes of comparison. Moreover, previous cadaveric studies conducted in our laboratory using similar digitization techniques, including preliminary data collected from adult trapezius muscles ${ }^{[18]}$ have reported consistent patterns of muscle architecture between specimens. ${ }^{[19,20]}$ It is also worth noting the limitation presented by the use of embalmed cadaveric specimens. Neither tissue properties nor architectural parameters are directly 
comparable between cadaveric and in vivo imaging studies; ${ }^{[36]}$ however, the use of proportional values will help facilitate comparisons between the data presented in this study and future in vivo studies. ${ }^{[3]}$

Despite a small sample size, we strongly believe that these preliminary results warrant further investigation into the changing morphometry of trapezius throughout post-natal development and adulthood. While longitudinal studies are not possible in cadaveric research, patterns of morphometry between specimens at different developmental timepoints have the potential to provide valuable insights into how muscles adapt to functional demands and how structure is associated with pathologic conditions. Likewise, a better understanding of the underlying anatomy of trapezius will provide an essential foundation upon which to create new imaging protocols for the collection of in vivo longitudinal developmental data. ${ }^{[37]}$ Future studies should focus on collecting detailed anatomical data to better understand the internal structure of trapezius, ideally from a range of development timepoints.

\section{Conclusion}

This is the first pilot study to digitize and quantify the complete $3 \mathrm{D}$ architecture of the infant and adult trapezius and offer preliminary evidence of how the morphology and architecture of trapezius differ between infancy and adulthood. Literature describing the detailed anatomy of trapezius is sparse, particularly before adulthood, leaving a notable gap in our understanding of how structure informs function during post-natal development. Future longitudinal studies should build upon the findings presented in this paper to document the post-natal developmental trajectory of trapezius using in vivo imaging techniques.

\section{Acknowledgements}

We would like to acknowledge and thank the individuals who donated their bodies for the advancement of education and research through the University of Toronto Willed Body Program. We would also like to thank William Wood and Harun Bola for their valuable technical assistance.

\section{Conflict of Interest}

The authors have no conflicts of interest.

\section{Author Contributions}

MLS: project development, data collection \& analysis, manuscript writing \& editing; LRB: data collection \& analysis, manuscript editing; EMB: data collection \& analysis, manuscript editing; AMRA: project development, manuscript writing \& editing; SAM: project development; manuscript editing.

\section{Ethics Approval}

Ethics approval was obtained from the Research Ethics Board at the University of Toronto (\#32679 \& \#27210). Donor consent was obtained in accordance with the Anatomy Act and The Trillium Gift of Life Network Act of the Province of Ontario, Canada.

\section{Funding}

The study was funded by Canadian Institute of Health Research (CIHR) Vanier Canada Graduate Scholarship [MLS].

\section{References}

1. Moore KL, Dalley AF, Agur AMR. Clinically oriented anatomy. 8th ed. Philadelphia: Lippincott Williams \& Wilkins; 2018. 1168 p.

2. Lindman R, Hagberg M, Bengtsson A, Henriksson KG, Thornell LE. Changes in trapezius muscle structure in fibromyalgia and chronic trapezius myalgia. In: Jacobsen S, Danneskiold-Samsoe B, Lund B, editors. Musculoskeletal pain, myofascial pain syndrome and the fibromyalgia syndrome. New York (NY): Haworth Medical Press; 1993. p. 171-6.

3. Olsen NJ, Park JH. Skeletal muscle abnormalities in patients with fibromyalgia. Am J Med Sci 1998;315:351-8.

4. Boyd RN, Morris ME, Graham HK. Management of upper limb dysfunction in children with cerebral palsy: a systematic review. Eur J Neurol 2001;8(Suppl 5):150-66.

5. Tasca G, Monforte M, Iannaccone E, Laschena F, Ottaviani P, Leoncini E, Boccia S, Galluzzi G, Pelliccioni M, Masciullo M, Frusciante R, Mercuri E, Ricci E. Upper girdle imaging in facioscapulohumeral muscular dystrophy. PLoS One 2014;9: e100292.

6. Powers SK, Lynch GS, Murphy KT, Reid MB, Zijdewind I. Disease-induced skeletal muscle atrophy and fatigue. Med Sci Sports Exerc 2016;48:2307-19.

7. Badura M, Grzonkowska M, Baumgart M, Szpinda M. Quantitative anatomy of the trapezius muscle in the human fetus. Adv Clin Exp Med 2016;25:605-9.

8. Wallden M. The trapezius-clinical \& conditioning controversies. J Body Mov Ther 2014;18:282-91.

9. Thelen E, Spencer JP. Postural control during reaching in young infants: a dynamic systems approach. Neurosci Biobehav Rev 1998;22:507-14.

10. Elliott JM, Jull GA, Noteboom JT, Durbridge GL, Gibbon WW. Magnetic resonance imaging study of cross-sectional area of the cervical extensor musculature in an asymptomatic cohort. Clin Anat 2007;20:35-40.

11. Stemper BD, Baisden JL, Yoganandan N, Pintar FA, Paskoff GR, Shender BS. Determination of normative neck muscle morphometry using upright mri with comparison to supine data. Aviat Space Environ Med 2010;81:878-82.

12. Debernard L, Robert L, Charleux F, Bensamoun SF. Characterization of muscle architecture in children and adults using magnetic resonance elastography and ultrasound techniques. J Biomech 2011;44:397-401. 
13. Adigozali H, Shadmehr A, Ebrahimi E, Rezasoltani A, Naderi F. Ultrasonography for the assessment of the upper trapezius properties in healthy females: a reliability study. Muscles Ligaments Tendons J 2016;6:167-72.

14. Mayoux-Benhamou MA, Barbet JP, Bargy F, Vallée C, Revel M. Method of quantitative anatomical study of the dorsal neck muscles. Surg Radiol Anat 1990;12:181-5.

15. Johnson G, Bogduk N, Nowitzke A, House D. Anatomy and actions of the trapezius muscle. Clin Biomech 1994;9:44-50.

16. Kamibayashi LK, Richmond FJ. Morphometry of human neck muscles. Spine 1998;23:1314-23.

17. Borst J, Forbes PA, Happee R, Veeger D. Muscle parameters for musculoskeletal modelling of the human neck. Clin Biomech 2011; 26:343-51.

18. Stiver ML, Käärid K, Kumbhare D, Agur AM. Comprehensive 3D architecture of the adult human trapezius: a cadaveric study. The FASEB Journal 2019;33:77.2-77.2.

19. Kim SY, Boynton EL, Ravichandiran K, Fung LY, Bleakney R, Agur AM. Three-dimensional study of the musculotendinous architecture of supraspinatus and its functional correlations. Clin Anat 2007;20: 648-55.

20. Fung L, Wong B, Ravichandiran K, Agur A, Rindlisbacher T, Elmaraghy A. Three-dimensional study of pectoralis major muscle and tendon architecture. Clin Anat 2009;22:500-8.

21. Agur AM, Ng-Thow-Hing V, Ball KA, Fiume E, McKee NH. Documentation and three-dimensional modelling of human soleus muscle architecture. Clin Anat 2003;16:285-93.

22. Lee D, Ravichandiran K, Jackson K, Fiume E, Agur A. Robust estimation of physiological cross-sectional area and geometric reconstruction for human skeletal muscle. J Biomech 2012;45:1507-13.

23. Hamilton WJ. Textbook of human anatomy. 2nd ed. London: Macmillan; 1956.753 p.

24. Gray H. Anatomy of the human body. 20th ed. Philadelphia (PA): Lea\&Febiger; 1918. 1396 p.

25. Grant JCB. The Musculature. In: Schaeffer JP, editor. Morris' human anatomy. 10th ed. Philadelphia (PA): The Blakiston Company; 1942. p. 377-581.

ORCID ID:

M. L. Stiver 0000-0002-8045-7291; L. R. Bradshaw 0000-0002-8722-8440; E. M. Breinhorst 0000-0002-7535-1361; A. M. R. Agur 0000-0002-2303-3628; S. A. Mirjalili 0000-0002-1599-3573
26. Holtermann A, Roeleveld K, Mork PJ, Grönlund C, Karlsson JS, Andersen LL, Olsen HB, Zebis MK, Sjøgaard G, Søgaard K. Selective activation of neuromuscular compartments within the human trapezius muscle. J Electromyogr Kines 2009;19:896-902.

27. Zanca GG, Oliveira AB, Ansanello W, Barros FC, Mattiello SM. EMG of upper trapezius-electrode sites and association with clavicular kinematics. J Electromyogr Kines 2014;24:868-74.

28. Reighard J, Jennings H. Anatomy of the cat. New York (NY): H. Holt and Company; 1901. 498 p.

29. Van Ee CA, Nightingale RW, Camacho DL, Chancey VC, Knaub KE, Sun EA, Myers BS. Tensile properties of the human muscular and ligamentous cervical spine. Stapp Car Crash J 2000;44:85-102.

30. O'Sullivan C, Meaney J, Boyle G, Gormley J, Stokes M. The validity of rehabilitative ultrasound imaging for measurement of trapezius muscle thickness. Man Ther 2009;14:572-8.

31. Li F, Laville A, Bonneau D, Laporte S, Skalli W. Study on cervical muscle volume by means of three-dimensional reconstruction. J Magn Reson Imaging 2014;39:1411-6.

32. Cutts A. Shrinkage of muscle fibres during the fixation of cadaveric tissue. J Anat 1988;160:75-8.

33. Cutts A, Seedhom BB. Validity of cadaveric data for muscle physiological cross-sectional area ratios: a comparative study of cadaveric and in-vivo data in human thigh muscles. Clin Biomech 1993;8:15662 .

34. Gerber RJ, Wilks T, Erdie-Lalena C. Developmental milestones: motor development. Pediatr Rev 2010;31:267-77.

35. Basmajian JV, De Luca CJ. Muscles alive: their functions revealed by electromyography. 5th ed. Baltimore (MD): Williams \& Wilkins; 1985. $561 \mathrm{p}$.

36. Martin DC, Medri MK, Chow RS, Oxorn V, Leekam RN, Agur AM, McKee NH. Comparing human skeletal muscle architectural parameters of cadavers with in vivo ultrasonographic measurements. J Anat 2001;199:429-34.

37. Kim S, Bleakney R, Boynton E, Ravichandiran K, Rindlisbacher T, McKee N, Agur A. Investigation of the static and dynamic musculotendinous architecture of supraspinatus. Clin Anat 2010;23:48-55.

Correspondence to: Mikaela Stiver, MSc, PhD(c)

Division of Anatomy, Department of Surgery, Faculty of Medicine,

University of Toronto, Toronto, Ontario, Canada

Phone: +1 2269793366

e-mail: mikaela.stiver@mail.utoronto.ca

Conflict of interest statement: No conflicts declared.

This is an open access article distributed under the terms of the Creative Commons Attribution-NonCommercial-NoDerivs 4.0 Unported (CC BY-NCND4.0) Licence (http://creativecommons.org/licenses/by-nc-nd/4.0/) which permits unrestricted noncommercial use, distribution, and reproduction in any medium, provided the original work is properly cited. How to cite this article: Stiver ML, Bradshaw LR, Breinhorst EM, Agur AMR, Mirjalili SA. Threedimensional muscle architecture of the infant and adult trapezius: a cadaveric study. Anatomy 2021;15(1):26-35. 